\title{
The Role of Probiotics in Inflammatory Responses of Critically-Ill Burn Patients (A Randomized Clinical Trial)
}

\author{
Oki Nugraha Putra ${ }^{1 *}$, Hengky Pebrianton², Suharjono ${ }^{3}$, Iswinarno DS ${ }^{4}$, Dwi Rahayu R \\ ${ }^{1}$ Study Program of Pharmacy, Faculty of Medicine, Hang Tuah University, Surabaya \\ ${ }^{2}$ Clinical Pharmacy of Mintohardjo Hospital, Jakarta \\ ${ }^{3}$ Departement of Clinical Pharmacy, Faculty of Pharmacy, Airlangga University, Surabaya \\ ${ }^{4}$ Department of Plastic Reconstructive and Aesthetic Surgery, Dr. Soetomo Hospital, Surabaya \\ ${ }^{5}$ Clinical Pharmacy of Dr. Soetomo Hospital, Surabaya \\ *Corresponding author: okinugrahaputra@yahoo.com
}

\begin{abstract}
Background: Impairment of intestinal barrier function and increased translocation of bacteria to the systemic blood flow contribute to the emergence of sepsis in burn patients. Oral probiotic administration has been advocated for treatment and prevention of diverse range of infection disease by modulating the host immune reponse. Objectives: This research was to determine the effect of probiotics containing Lactobacillus and Bifidobacterium on leucocyte and neutrophil level and on the clinical outcome in burn patients. Methods: Seventeen burn patients with burns involving more than $15 \%$ total body surface area were randomized in a prospective, double-blind, non-placebo, assigned to receive mono and multistrain probiotics (Lactobaccilus and Bifidobacteria) once daily for consecutive fourteen days. Laboratory measurements of Leucocyte and Neutrophil were done on days 4 dan 19. Clinical outcomes including incidence of diarrhea, infection, and mortality were also recorded. Results: There was no incidence of diarrhea and incidence of sepsis and also mortality were similiar between two groups during this study. Multistrain probiotics reduced significant level both of leucocytes and neutrophil during this study $(P<0,05)$ with significant differences between two groups only in neutrophil level. Conclusion: Administration of monostrain and multistrain probiotics to burn patients caused better clinical outcomes and reduced level of inflammatory parameters (leucocyte and neutrophil). It might be considered as an adjunctive therapy in the treatment of burn patients to treat infection.
\end{abstract}

Keywords: Probiotics, Burn Patients, Leucocyte, Neutrophil

\section{INTRODUCTION}

Critically ill burn patients are more susceptible than other critically ill patients to acquire infections as traditionally reported in infection surveillance systems in the Intensive Care Units (ICU) (Dudeck et al., 2003). Another characteristic to resolve infections in burn patients is the low value of clinical criteria, i.e.fever, and biomarkers to differentiate systemic inflammatory response syndrome (SIRS) from sepsis (Greenhalgh et al., 2007). The severe burn patient, i.e. burns $>20 \%$ of the body surface in adults usually shows signs of inflammation without a proven infection. This difficulty differentiating inflammation form infection can lead in some cases to excessive use of antibiotics with associated costs and the possibility to select resistant flora. On the other hand delay in the administration of appropriate antibiotics may be associated with increased morbidity and mortality (Hidalgo et al., 2016). 
Although the inflammation is initiated almost immediately after the burn injury, the systemic response progresses with time, usually peaking 5 to 7 days after the burn injury (De la Cal MA et al., 2005). Much of the local and certainly the majority of the distant changes are caused by inflammatory mediators (Lars et al., 2010). Thermal injury initiates systemic inflammatory reactions producing burn toxins and oxygen radicals and finally leads to peroxidation. Natural resistance to infection in traumatic wounds is predominantly a function of the innate immune system. Following thermal injury, the innate immune system responds immediately by stimulating localized and systemic inflammatory reactions. The innate immune response participates in activating the adaptive immune response; however, in so doing it has an adverse affect on the burn victim's ability to mount a vigorous immune response to invading microorganisms and, therefore, predisposes the burn victim to infectious complications. The innate immune system itself is composed of natural barriers to microbial invasion as well as cellular (leucocyte) and humoral (complement) elements (D’Arpa et al., 2007)

Gastrointestinal (GI) tract has been blamed for the pathogenesis of sepsis and MODS due to the impairment of intestinal barrier function and increased translocation of bacteria to systemic blood flow (MacFie et al., 1999; Hassoun et al., 2001). Various treatment modalities were investigated to prevent the occurrence of bacterial translocation and enhance immune function after thermal injury (Kurmis et al., 2010). One such treatment involves a supplementation of probiotics. Probiotics are defined according to the World Health Organization (WHO) as "live micro-organisms which, when administered in adequate amounts, confer a health benefit on the host" (Food and Health Agriculture Organization of United Nations and World Health Organization, 2002). Major strains of probiotics include Lactobacillus and Bifidobacterium species.

Probiotics bacteria also influence the immunological defense system by the production of diverse cytokines that enhance the reaction of the immunological system to pathogens, enhancement of the phagocytic capabilities of polymorphonuclears , augmentation of natural killer cell activity, and amplification of production of specific antibodies against pathogenic bacteria (Maldonado et al., 2007). Augmentation of the gut barrier with the use of probiotics has been observed in burn rat models (Wang et al., 2003; Zhang and Shi, 2014). Nevertheless, the probiotic effect has not been thoroughly examined in the clinical burn setting. Thus, the purpose of this double-blind, non placebo-controlled, and randomized clinical trial was to determine the effect of mono and multistrain probiotics containing Lactobacillus, Bifidobacterium and Streptococcus thermophilus on leucocyte dan neutrophil level and on the clinical outcome in burn patients.

\section{METHOD}

This was a 14-day, close label, double blind, randomized, parallel-group study, single centre, conducted in the burn unit of Dr. Soetomo Hospital (Surabaya, East Java, Indonesia) between June 2016 and October 2016. After approval of ethics committee of Dr. Soetomo Hospital and obtaining informed consent of the patients or their legal, twenty three patients were enrolled in this study. The study protocol was approved by commitee ethics of Dr.Soetomo. Due to the unconsciousness of patients, informed consent was obtained form their closest family members prior to their admittance into the study.

The following inclusions criteria were critically burn patients : aged 18-60 years old, extensive burns more than $15 \%$ total body surface with minimal grade IIAB that occurred less than 24 hours after the event, could be fed orally or enterally within of hospital admission, and willing to follow the study by signing a letter of approval. Exclusion criteria were inability to be fed via the gastrointestinal tract, patient had received probiotics before admitted to the study, those with previous or current gastrointestinal disease, or with chronic disease such as diabetes, 
sepsis when admitted to the hospital, pregnancy and lactating, immune disorders and patients who were expected to expire in the next 24 hours.

After inclusion (which was done within 72 hours after admission), patients were randomized with a ratio of 1:1 into once daily oral treatment with monostrain or multistrain probiotic. Randomization was done with a simple random number and kept in a remote secure location and administrated by an independent third party who was not involved with the clinical conduct of the study until all study data were collected and verified. Patients and those involved in enrolling participants, administering interventions and assessing outcomes were blind to group assignments.

Both of treatment groups received one capsule of viable bacteria at $10.00 \mathrm{pm}$. Monostrain probiotics contained $>10^{7} \mathrm{cfu}$ Lactobacillus acidophilus and Bifidobacterium longum and multistrain probiotics contained $>10^{8} \mathrm{cfu}$ viable lyophilized bacteria consisting of four strains of Lactobacillus (Lactobacillus casei, Lactobacillus rhamnousus, Lactobacillus acidophilus, and Lactobacillus delbrueckii subsp. Bulgaricus), two strains of Bifidobacterium (Bifidobacterium longum, Bifidobacterium breve) and Streptococcus salivarius subsp. Thermophilus. If the patient transitioned to parenteral nutrition and could not resume enteral feeding for more than 2 days, the study was discontinued prematurely.

Probiotic was provided to enrolled patients within 4 days after the begining of burns and for 14 consecutive days. Both of dosage form of probiotics were dispensed in identical packaging by pharmacy departement. The probiotics were given orally, dissolved in sufficient distilled water and administered through a nasogastric tube. When patients started oral intake and the nasogastric tube was removed, probiotics were ingested orally for the remaining days. Disallowed concomitant medications included any other product containing probiotics, prebiotics during the study. Another standard therapy for these patients included operation when necessary such as antibiotics and analgesics.

The primary endpoint was the leucocyte and neutrophil level before and after 14 days of probiotics treatment. Secondary endpoints included gastrointestinal tract tolerance (incidence of diarrhea), incidence of infection (burn-related or systemic sepsis) and mortality. Both of endpoints were recorded and compared between two groups

Blood sample was obtained from burn patients to measure leucocyte and neutrophil level on days 4 (before initiation of probiotics) and days 19. Leucocyte and neutrophil were measured by Automated hematology analyzer (flow cytometry) in the laboratory of clinical pathology, Dr. Soetomo Hospital, Surabaya.

The total sample size of subjects was calculated based on published levels of leucocyte differences in burn patients. Samples were selected from burn center of Dr.Soetomo Hospital, Surabaya using convenience sampling method. Kolmogorov-Smirnov test was used to assess whether continuous data were normally distributed. For continuous variables, differences between groups were tested with Student's $t$ tests or analysis of variance for normally distributed data or the Mann-Whitney $U$ test for non-normally distributed data. Comparisons of categorical data were done by chi-squared test or Fisher's exact test. Two-sided $P<0.05$ was considered statistically significant. The data was analyzed using statistical software program Statistical Package for the Social Science (SPSS) version 16.0.

\section{RESULTS AND DISCUSSION}

During June 2016 - October 2016, there were twenty three burn patients who fulfilled the inclusion criteria to be randomized. Six patients died during fourteen days treatment period and can not complete the study or drop out. Seventeen patients who completed the study, eight in monostrain and nine in multistrain probiotics. Demographic characteristics of patients are 
shown in Table 1. No significant differences in age, sex, total body surface are (TBSA), inhalation injury and 14-day mortality.

As regards clinical evaluation, two patients in each groups developed sepsis during their hospital stay and there was not a significant difference between two groups $(P=0,637)$. All patients between two groups received antibiotics by intravenous during treatment period, and the highest percentage was ceftazidime. No significant difference was observed in terms of the days and total dose of cetazidime between two groups $(P>0,05)$ as shown in table II. No adverse effects of probiotic therapy were noted at any time during the study. No patients developed Lactobacillus-induced sepsis between two groups.

As expected, in both two groups levels of leucocyte and neutrophil were higher than those of normal human leucocyte and neutrophil on day 4 post admision. On day 19, the patients who received of both probiotics showed lower levels of leucocyte and neutrophil as shown in table III.

Table 1 Comparation of demographic and primary clinical status

\begin{tabular}{lccc}
\hline Variable & Mono Strain $(\mathrm{n}=11)$ & Multi Strain $(\mathrm{n}=12)$ & $P$ \\
\hline Sex (M:F) & $4: 7$ & $3: 9$ & $0,575^{*}$ \\
Age (y) & $41,6 \pm 13,7 * * *$ & $40,11 \pm 13,3$ & $0,763^{* *}$ \\
TBSA (\%) & $41,0 \pm 22,2 \%$ & $46,6 \pm 28,1 \%$ & 0,913 \\
Inhalation Injury & 5 & 6 & 0,488 \\
14-day mortality & 3 & 3 & 0,907
\end{tabular}

$*$ :chi square, $* *$ independent $t$-test, $* * *$ mean $\pm \mathrm{SD}, \mathrm{TBSA}=$ total body surface are, $\mathrm{SD}=$ standard deviation

Table 2 Clinical outcome data at the end of the study

\begin{tabular}{lccc}
\hline Variable & Mono Strain $(\mathrm{n}=8)$ & Multi Strain $(\mathrm{n}=9)$ & $P$ \\
\hline Sepsis (n.\%) & $2(25 \%)$ & $2(22 \%)$ & $0,637^{*}$ \\
$\begin{array}{l}\text { Duration of } \\
\text { (days) }\end{array}$ & $10,0 \pm 3,9 * *$ & $8,5 \pm 5,0$ & 0,537 \\
Total dose of ceftazidime & $30,1 \pm 11,3$ & $24,6 \pm 15,4$ & 0,452 \\
Diarrhea (n.\%) & $0(0 \%)$ & $0(0 \%)$ & 0,906 \\
\hline
\end{tabular}

*independent $t$-test, **mean \pm SD

Table 3 Leucocyte and neutrophil level during the intervention period

\begin{tabular}{lcccc}
\hline Variable & Day & Mono Strain $(\mathrm{n}=8)$ & Multi Strain $(\mathrm{n}=9)$ & $P$ \\
\hline Leucocyte $\left(10^{3} / \mathrm{mm}^{3}\right)$ & 4th day & $16,90 \pm 4,98$ & $18,07 \pm 7,14^{*}$ & $0,705^{ \pm}$ \\
& 19th day & $15,68 \pm 6,27$ & $11,88 \pm 4,90$ & 0,189 \\
Neutrophil $(\%)$ & 4th day & $86,3 \pm 6,3$ & $87,6 \pm 6,9$ & 0,709 \\
& 19th day & $87,01 \pm 5,9$ & $79,58 \pm 3,9$ & 0,026 \\
\hline
\end{tabular}

*mean \pm SD $\quad \pm$ independent samples- $t$ test 


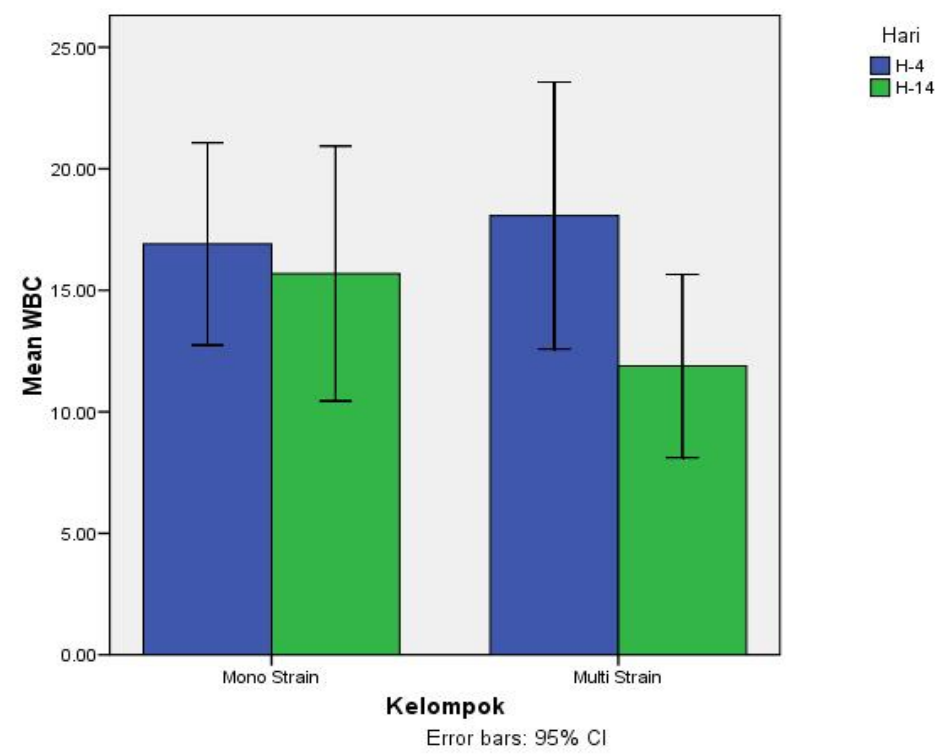

Figure 1 Mean \pm SD leucocyte level in two groups. Patients in the multistrain probiotic group had significanty lower of leucocyte level by day $14 .(P=0,044)$

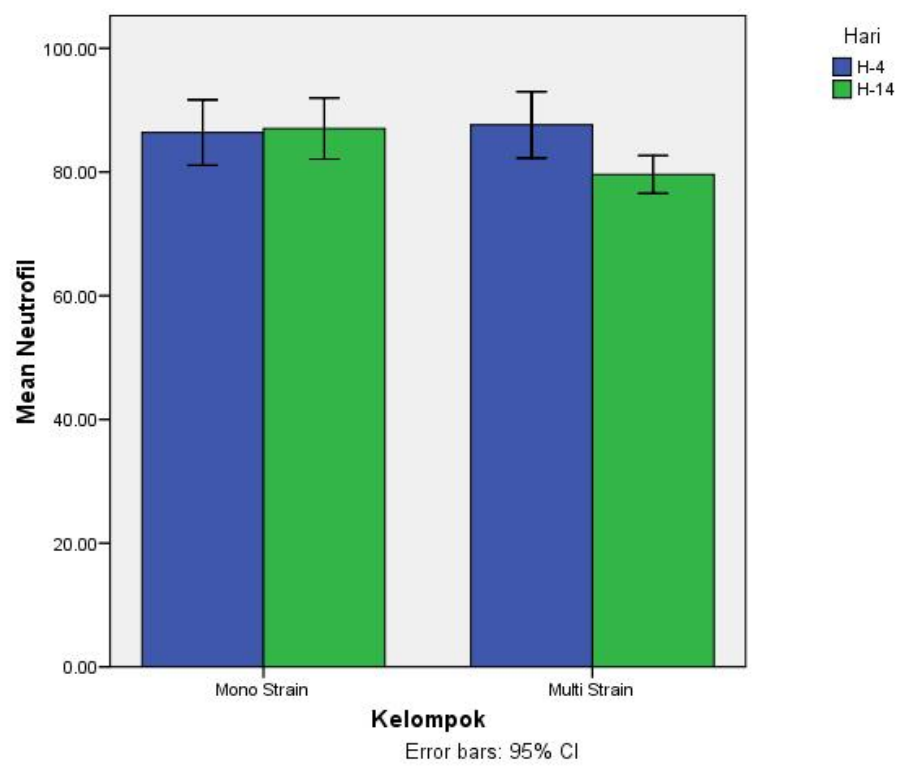

Figure 2 Mean \pm SD leucocyte level in two groups. Patients in the multistrain probiotic group had significanty lower of neutrophil level by day $14 .(P=0,011)$

Multiple studies have evaluated probiotic consumption in humans and concluded that probiotics are safe and can confer health benefits, such as disease treatment and prevention; however, there is a lack of evidence regarding the benefits of probiotics for patients with critical illness, such as burns (Andrea et al., 2009). The present study used a double-blind, non placebo and randomized design to determine the effect of probiotic supplementation (monostrain and multistrain) on the outcome of adult patients after burn injuries.

Burn patients are at very high risk of developing hospital acquired infection that may lead to multiple organ failure and impair prognosis. Several experimental and clinical studies suggest that an inexpensive and easy to use treatment, namely enteral probiotics administration, could be effective in the ICU by improving immune competency, reducing oro-pharyngeal 
bacterial colonization, and even nosocomial infections (Saavedra, 2001; Isakow et al., 2007; Watkinson et al., 2007). Seventeen acutely-burned patients were included in this study. Subjects were randomly allocated into two equal groups. Demographically, there was no significant difference between the two groups .

In our study, there was a small number in the incidence of sepsis between two groups with no significant result $(\mathrm{p}=0,637)$. Our data confirmed the findings of Morrow et al, 2010 (Morrow et al., 2010) who evaluated the use of probiotics in ICU patients, and Kotzampassi et al (2006), who investigated the effect of synbiotics on post-traumatic critically ill patients. They found that there was a significant reduction in rate of infection, incidence of ventilator associated pneumonia (VAP) and mortality. In another study Jebur et al (2010), found that Lactobacillus acidophilus at a concentration of $1 \times 10^{8}$ cells $/ \mathrm{ml}$ (in vitro) can inhibit the growth of all gram positive and gram negative pathogenic bacteria that can infect burn wounds.

Importantly, we observed that there was no incidence of diarrhea between two groups which is consistent with the results of Frohmader et al. (2010), who described the effectiveness of probiotics in reducing liquid stool frequency in critically ill patients. Similar benefits of probiotic therapy were reported in a meta-analysis that evaluated 25 randomized controlled trials and concluded that probiotics can reduce the frequency of diarrhea and the incidence of Colostridium difficile infection associated with antibiotic use (McFarland, 2006). However, another recent report on the impact of probiotics in critically ill patients showed they had no effect on incidence of diarrhea (Siempos et al., 2010). One hypothesis for this could be that probiotic effect depends on the type of strain and the site of action; for example, strains acting primarily at the small intestine confer different effects than those which act primarily on the large intestine, with the greatest impact on diarrhea expected to occur from the colonic strains.

Leucocytosis and neutrophilia are occured in both treatment groups on 4 days after burns. Eventough leucocyte is influenced by many non infection factors such as infarc myocard, catecholamine, corticosteroid and acute bleeding in burn injuries, leucocyte now is still used as a routine blood evaluation for diagnostic marker in infection or even sepsis. Leucocytosis is a normal inflammation response of the body related to burn. Inflammation occured immediately after burn and accompanied by higher level of leucocyte (Fayazof et al., 2009). Our result is similar to Stoilova et al. (2007), who studied the effect of burn in immunological parameter of burn patients and demonstrated a significant rise in leucocyte in 7 days after burn compared with control. Our data are also consistent with those of Alberda et al. (2007) who studied the effect of probiotic therapy in critically ill patients and demonstrated those patients who received viable probiotics had a lesser decline in CRP concentrations than did those patients who received either placebo or bacterial sonicates (Alberda et al, 2007). CRP, commonly used as a marker of systemic inflammation (Ballow and Kushner, 1992), is an acute-phase protein produced by the liver and by endothelial cells (Cesari et al, 2003). CRP inhibits the production of proinflammatory cytokines and chemokines, including tumor necrosis factor and interferon $\gamma$ (Szalai et al., 2002) and also demonstrates significant antimicrobial activity (Szalai, 2002).

Multistrain probiotics reduced lower level of leucocyte than monostrain's. In intestine, a protein molecule named heat shock protein (hsp), hsp25 and hsp72, keep tight junction in epithelia cells and gut barier. Multistrain probiotics may stimulate hsp25 and hsp27, so that translocation of pathogenic bacteria or lipopolysaccharide from intestine to systemic circulation which stimulate inflammation response can be reduced (Carissa and James, 2010). Multistrain probiotics will produce much more short chain fatty acids (propionic acid, acetic acid and butiric acid) from carbohydrate metabolism. Butiric acid acts an important role as inflammatory modulation with antiinflammation effect in intestinal epitel cells, macrophage and leucocyte. Butiric acid also acts as carrier signal molecule to inhibit NFKB for releasing proinflammation cytokines in different mechanims (Noha et al., 2006). 
Neutrophils, which are a type of polymorphonuclear leukocyte, are well recognized as one of the major players during acute inflammation. They are typically the first leukocytes to be recruited to an inflammatory site and are capable of eliminating pathogens by multiple mechanism. Data from experimental and clinical settings show that, following infection, the localization of neutrophils to the site of inflammation is crucial for clearance of the infection. Our result showed both of treatment groups had a higher level of neutrophil (neutrophilia). Our results are consistent with those of Stoilova et al. (2017), who studied the effect of burn injury in immunology and microbiology parameters. It demonstrated a lower phagocytic index compared with control. Phagocytic index is persentage of polymorphonuclear leukocytes (PMN), one of them is neutrophil to bacterial phagocytosis. Besides, segmented leucocytes (neutrophil) was higher than control however the result is not significant. Neutrophil extracellular traps (NETs) are an essential component of the antimicrobial repertoire and represent an effective means by which neutrophils capture, contain, and kill microorganisms. However, the uncontrolled or excessive liberation of NETs also damages surrounding cells and can contribute to disease pathophysiology (Brinkmann et al., 2004). In multistrain probiotics, the neutrofil level was significant decrease at the end of the study. Our result was consistent by Linda et al. (2014) which demonstrated that probiotic Lactobacillus rhamnosus strain GG inhibits both PMA- and Staphylococcus aureus -induced formation of NETs. It concluded that probiotic bacteria, LGG as an inhibitor of NETosis.

\section{CONCLUSIONS}

Both mono and multistrain probiotics can effectively enhance immune function by significantly reduce the level of leucocyte and neutrophil, however the significant differences only in neutrophil level between two groups. No adverse event of probiotics observed during the study.

\section{ACKNOWLEDGEMENTS}

Authors would like to thank all staff at the burn center of Dr. Soetomo hospital for their corporation during the study, and the laboratory of clinical pathology of Dr. Soetomo hospital for the support in Automated hematology analyzer (flow cytometry)

\section{REFERENCES}

A.D. Fayazov., S.I. Shukourov., B.I. Shukurov, et al. (2009). Disorders of the immune system in severly burned patients. Annals of Burns and Fire Disasters (3), 121-130.

Alberda, C., Gramlich, L., Meddings, J., Field, C., McCargar, L. Kutsogiannis, D, et al. (2007). Effects of probiotic therapy in critically ill patients: A randomized, double-blind, placebo-controlled trial. Am J Clin Nutr, 85, 816-823.

Andrea, T. B., Carlo, S., et al. (2009). Probiotics and Immcentery. Journal of Gastroenterology(44), 26-46.

Ballou, S.P., \& Kushner, I. (1992). C-reactive protein and the acute phase response. Adv Intern Med, 37. 313-36.

Brinkmann, V., U. Reichard, C. Goosmann, B. Fauler, Y. Uhlemann, D. S. Weiss, Y. Weinrauch, and A. Zychlinsky. (2004). Neutrophil extracellular traps kill bacteria. Science 303, 1532-1535

C. Maldonado Galdeano., A. De Moreno de LeBlanc., et al. (2007). Proposed Model: Mechanisms of immunomodulation induced by probiotics bacteria, clinical and vaccine immunology. American Society For Microbiology, 485-492 
Carissa, M.T., \& James, V. (2010). Probiotics-host communication, modulation of signaling pathways in the intestine. Gut Microbes, 1(3), 148-163.

Cesari, M., Penninx, B.W., Newman, A.B., et al. (2003). Inflammatory markers and cardiovascular disease (The Health, Aging and Body Composition [Health ABC] Study). Am J Cardiol, 92, 522- 8.

D’Arpa A, Accardo-Palumbo A, Amato G, D'Amelio L, Napoli B, Pileri D, et al. (2007). Decrease of circulating dendritic cells in burn patients. Ann Burns Fire Disasters, 20(4), 199-202.

De la Cal MA, Cerdá E, García-Hierro P, van Saene HK, Gómez- Santos D, Negro E, et al. (2005). Survival benefit in critically ill burned patients receiving selective decontamination of the digestive tract: a randomized, placebo-controlled, double-blind trial. Ann Surg, 241, 424---30.

Dudeck, M.A., Weiner, L.M., Allen-Bridson, K., Malpiedi, P.J., Peterson, K.D., Pollock, D.A., et al. (2003). National Healthcare Safety Network (NHSN) report, data summary for 2012, device-associated module. Am J Infect Control, 41, 1148---66.

F. Hidalgo, D. Mas, M. Rubio, P.Garcia-Hierro. (2016). Infection in critically ill burn patients. Medicina Intensiva, 40(3), 179-185.

Food and Health Agriculture Organization of United Nations and World Health Organization. Guidelines for the evaluation of probiotics in food. (2002). Joint FAO/WHO Working Group Report on Drafting Guidelines for the Evaluation of Probiotics in Food

Frohmader, T.J., Chaboyer, W.P., Robertson, I.K., et al. (2010). Decrease in frequency of liquid stool in enterally fed critically ill patients given the multispecies probiotic VSL\#3: a pilot trial. Am J Crit Care, 19, e1-11.

Greenhalgh, D.G., Saffle, J.R., Holmes, J.H., Gamelli, R.L., Palmieri, T.L.,.Horton, J.W., et al. (2007). American Burn Association consensus conference to define sepsis and infection in burns. J Burn Care Res, 28, 776---90.

Hassoun, H.T., Kone, B.C., Mercer, D.W., Moody, F.G., Weisbrodt, N.W., Moore, F.A. (2001). Post-injury multiple organ failure: The role of the gut. Shock, 15, 1-10.

Isakow, W., Morrow, L.E., Kollef, M.H. (2007). Probiotics for preventing and treating nosocomial infections: review of current evidence and recommendations. Chest, 132, 286-29.

Jebur, M.S. (2010). Therapeutic efficacy of lactobacillus acidophilus against some bacterial isolates burn wound cases. North Am J Med Sci, 2, 586- 91.

Kotzampassi, K., Giamarellos-Bourboulis, E.J.,Voudouris, A., Kazamias P, Eleftheriadias E. (2006). Benefits of a synbiotic formula (Synbiotic 2000 Forte) in critically ill trauma patients: early results of a randomized controlled trial. World J Surg, 30, 1848-55.

Kurmis, R., Parker, A., \& Greenwood, J. (2010). The use of immunonutrition in burn injury care: Where are we? J Burn Care Res, 31, 677-91.

Lars, H. E., Dhaval, B., \& Peter, M. (2010). The biology of burn injury, john wiley \& sons A/S, Experimental Dermatology (19), 777-783.

Linda, V., Robert, J. L., Amit, A., Michael, G., Philip, M. S. (2014). Probiotic lactobacillus rhamnosus inhibits the formation of neutrophil extracellular traps. The Journal of Immunology, 192, 1870-1877.

MacFie, J., O’Boyle, C., Mitchell, C.J., Buckley, P.M., Johnstone, D., \& Sudworth, P.(1999). Gut origin of sepsis: A prospective study investigating associations between bacterial translocation, gastric microflora, and septic morbidity. Gut, 45, 223-8.

McFarland, L.V. (2006). Meta-analysis of probiotics for the prevention of antibiotic associated diarrhea and the treatment of Clostridium difficile disease. Am J Gastroenterol, 101, 812-22. 
Morrow, L.E., Kollef, M.H., Casale, T.B. (2010). Probiotic prophylaxis of ventilator associated pneumonia: a blinded, randomized, controlled trial. Am J Respir Crit Care Med, 182(8), 1058-64.

Noha, E. S., Helen, F.G., Ola, S.,Myriam, H. (2006). Delayed neutrophil apoptosis in patients with multiple organ dysfunction syndrome. Critical Care and Shock 9, 9-15.

Saavedra, J.M. (2001). Clinical applications of probiotic agents. Am J Clin Nutr, 73, 1147S$1151 \mathrm{~S}$

Siempos II, Ntaidou, T.K., Falagas, M.E. (2010). Impact of the administration of probiotics on the incidence of ventilator-associated pneumonia: A meta analysis of randomized controlled trials. Crit Care Med, 38, 954-62

Szalai, A.J., Nataf, S., Hu, X.Z., \& Barnum, SR. (2002). Experimental allergic encephalomyelitis is inhibited in transgenic mice expressing human C-reactive protein. J Immunol, 168, 5792-7.

Szalai, A.J. (2002). The antimicrobial activity of C-reactive protein. Microbes Infect, 4, 2015.

Wang, Z.T., Yao, Y.M, Xiao, G.X, Cao W.H, Sheng Z.Y. (2003). Bifidobacterial supplement enhances the expression and excretion of intestinal sIgA in severely burned rats. Zhonghua Wai Ke Za Zhi, 41, 385-8

Watkinson, P.J., Barber, V.S., Dark, P., \& Young, J.D. (2007). The use of pre-pro and synbiotics in adult intensive care unit patients: systematic review. Clin Nutr, 26, 182 192.

Stoilova Y.D, Haidushkal I.A, Murdjeval M.A, Traikov I.Z, Popova T.A, Kevorkyan A.K. (2007). Immunological and microbiological investigations of patients with burn injuries. Folia Med (Plovdiv),49(1-2), 49-58.

Zhang, Y.P., \& Shi, Z.R. (2004). Effects of probiotics on the bacterial groups of intestinal and sIgA in severely burned rats. Chinese Journal of Microecology, 16(5), 257-9. 